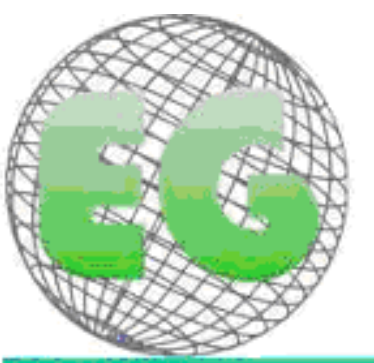

$N^{\circ} 18$
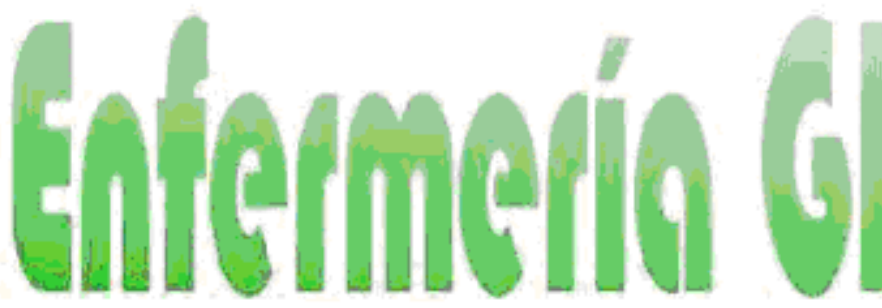

Revista electrônica cuatrimestral de Enfermeria

Febrero 2010

\title{
COMPETENCIAS PROFESIONALES DE LOS ENFERMEROS: EL MÉTODO DEVELOPING A CURRICULUM COMO POSIBILIDAD PARA ELABORAR UN PROYECTO PEDAGÓGICO
}

COMPETÊNCIAS PROFISSIONAIS DO ENFERMEIRO: O MÉTODO DEVELOPING A CURRICULUM COMO POSSIBILIDADE NA ELABORAÇÃO DE UM PROJETO PEDAGÓGICO

\section{${ }^{*}$ Chaves, MM, **Menezes Brito, MJ, ${ }^{* *}$ Cozer Montenegro, L, ${ }^{* * *}$ Alves, M. \\ *Mestre em Administração. **Doutora em Administração. Professora do Programa de Pós- Graduação. Vice-líder do Núcleo de pesquisa NUPAE/ CNPq. ***Mestranda em Enfermagem. Membro do Núcleo de Pesquisa NUPAE/CNPq. ****Doutora em Enfermagem. Professora do Programa de Pós-Graduação. Líder do Grupo de pesquisa NUPAE/ CNPq. Escola de Enfermagem da Universidad Federal de Minas Gerais (UFMG). Brasil.}

Palabras Clave: Enfermería, Educación en Enfermería, Formación Profesional, la competencia profesional Palabras Chave: Enfermagem, Educação em Enfermagem, Formação Profissional, Competência Profissional.

\section{RESUMEN}

El objetivo del presente estudio fue validar las competencias técnicas de los enfermeros para proporcionar ayudas para la construcción del plan de estudios de enseñanza del curso de enfermería de la Escuela de Enfermería de la Universidad Federal de Minas Gerais. Con este fin, hemos utilizado el método Developing a Curriculum (DACUM), que se configura como una técnica de enfoque cualitativo, por medio de la realización de un grupo focal, y que permite el levantamiento de las funciones, habilidades, actitudes y conocimientos de un trabajo en particular. Este estudio se llevó a cabo en 2005, con la participación de 18 enfermeras de diferentes especialidades que reúnen los siguientes criterios de inclusión: Tener 5 a 10 años de experiencia profesional en enfermería, estar empleadas exclusivamente en una función y ser considerada eficaz por otros profesionales. Como resultados percibimos las áreas de enfermería con una mayor tendencia de crecimiento en el mercado laboral, la caracterización del perfil de los enfermeros que trabajan en los servicios de Belo Horizonte y la identificación de las habilidades necesarias para la enfermera en diversas áreas de especialización. Esta metodología permitió desarrollar un proyecto de enseñanza de forma colectiva con la participación de profesores, estudiantes y enfermeras de los servicios integrando el servicio, la escuela y la enseñanza. 


\section{RESUMO}

Neste estudo buscou-se validar as competências técnicas dos enfermeiros a fim de fornecer subsídios para construção do currículo do curso de enfermagem da Escola de Enfermagem da Universidade Federal de Minas Gerais. Para tal, foi utilizado o método Developing a Currículum (DACUM), que se configura como uma técnica de abordagem qualitativa, baseada na realização de um grupo focal, e que permite o levantamento de funções, habilidades, atitudes e conhecimentos de um determinado cargo. Este estudo foi realizado no ano de 2005 e contou com a participação de 18 enfermeiros de diferentes especialidades que obedeceram aos seguintes critérios inclusão: ter de 5 a 10 anos de experiência profissional na enfermagem, estar empregado exclusivamente no cargo e ser considerado eficiente por seus pares. Como resultados pode-se perceber as áreas de enfermagem com maior tendência de crescimento no mercado de trabalho, caracterizamos o perfil dos enfermeiros que atuam nos serviços de Belo Horizonte e identificamos as competências necessárias para o enfermeiro em diversas áreas de atuação. Esta metodologia permitiu construir um Projeto Pedagógico de forma coletiva com a participação de professores, alunos e enfermeiros dos serviços integrando o ensino, o serviço e a escola.

\section{ABSTRACT}

The objective of this study was to validate the technical skills of nurses in order to provide subsidies for creation of nurse course curriculums for the Nursing School of Federal University of Minas Gerais, Brazil. To this end, we used the Developing a Curriculum method (DACUM), which sets up as a qualitative research technique, based on the creation of a focus group, and that allows identifying duties, skills, attitudes and knowledge of a particular job. This study was conducted in 2005, with 18 nurses from various specialties having the following inclusion criteria: Having 5 to 10 years of professional experience in nursing, being employed solely in a specific job and being considered efficient by other professionals. The results showed areas of nursing with a higher tendency of growth in the labor market, characterized the profile of nurses who work in the health services of Belo Horizonte, and identified the skills required of nurses in various specialized areas. This methodology allowed the development of a collective pedagogic project with the participation of teachers, students and nurses, integrating teaching, service and school.

\section{INTRODUCCIÓN}

Estos últimos años los Ministerios de Educación y de Salud han implementado estrategias articuladas para lograr cambios en el proceso formativo de los cursos de grado del área de salud. Los currículos de estos cursos deben adaptarse a formatos más actuales, basados en habilidades específicas y en competencias generales, asociados a un perfil profesional y humano para brindar educación orientada a los problemas de salud más serios de la población.

El debate sobre los cambios en la formación de los profesionales de salud, más apropiada a las necesidades sociales, ha sido reforzado mediante la propuesta de las Directrices Curriculares Nacionales para el Curso de Grado en Enfermería (DCNENF) que realzan la necesidad de formar profesionales competentes, críticos y comprometidos con la salud de la población ${ }^{(1)}$. Desde esta perspectiva, los gestores de la educación no han escatimado esfuerzos para construir colectivamente un Proyecto Pedagógico (PP) innovador y flexible. Algunas Facultades han sido incentivadas a adecuar sus currículos, privilegiando la integración entre saberes, conocimientos científicos de la universidad, sentido común y aspectos culturales del medio ${ }^{(2)}$.

En este escenario, durante las tres últimas décadas, la Escuela de Enfermería de la Universidad Federal de Minas Gerais-EEUFMG, ha llevado a cabo importantes 
transformaciones, elaborando instrumentos, métodos y principios involucrados no sólo en la trasmisión de conocimiento sino también en la formación sólida del alumno, preparándolo para enfrentar los retos de una sociedad en constante mutación.

De esta forma, la EEUFMG ha establecido como prioridad la construcción de un PP flexible adecuado a las DCNENF y a la nueva postura de la Universidad en lo referente a agilidad en la construcción del conocimiento. Para diseñar el nuevo currículo la estrategia ha sido solicitar la contribución de la comunidad académica y de los profesionales insertados en el mercado laboral. Se realza que la búsqueda de adherencia entre las DCNENF y los PP significa comprender la realidad del proceso de formación en enfermería en sus distintas concepciones, prácticas y desde la perspectiva de los distintos actores sociales. Preparar un profesional con el perfil que indican las DCNENF significa dotarlo de habilidades y competencias para actuar y pensar ante la diversidad y complejidad de los escenarios. El término competencia se inscribe en una matriz conceptual bastante amplia que también se emplea con frecuencia para designar competencias profesionales, conocimientos, aptitudes, capacidades, actitudes o como equivalente del saber-hacer y labor ${ }^{(3)}$.

El método Developing a Curriculum (DACUM) fue empleado con miras a considerar una estrategia que abarcase los distintos escenarios de actuación del enfermero durante el proceso curricular del curso de Enfermería de la EE-UFMG. Este método se originó en Canadá, fue desarrollado por Robert Adams y adoptado por la Holland College en 1970. Mediante un lenguaje patrón se identifican las exigencias de conocimientos, aptitudes, habilidades específicas y generales, destreza y actitudes solicitadas para un determinado cargo o conjunto de ocupaciones del mercado laboral ${ }^{(4)}$.

EI DACUM es una metodología empleada por muchos gobiernos y organizaciones para describir funciones y competencias de cargos, ocupaciones, desarrollar planes de estudio que sirvan de ayuda para la formación de mano de obra, planificación de carreras, selección de personas, implementación de modelos de competencias, entrenamiento y elaboración de cursos. Consiste en un modo eficaz de describir una determinada ocupación definiendo sus competencias profesionales que incluyen conocimientos, habilidades y actitudes. Esta metodología es eficiente por captar no solamente las actividades técnicas generalmente establecidas en los puestos de trabajo, sino también las habilidades de los profesionales en determinada función. EI DACUM presupone que el trabajador es la mejor persona para hablar sobre lo que hace, teniendo en cuenta que los atributos que se les exigen han suplantado las características técnicas establecidas en las descripciones de los cargos. Así, al analizar la función de los enfermeros por medio del DACUM, se crea lugar para la definición más consistente del registro de trabajo, ampliando las oportunidades de construir un plan de estudios integrado teniendo en cuenta la realidad de la situación social.

Considerando lo expuesto anteriormente, la propuesta del presente estudio es identificar, mediante la utilización del método DACUM, las grandes áreas de competencias profesionales en enfermería con miras a suministrar ayudas para la construcción del plan de estudios del curso de enfermería de la EEUFMG.

\section{METODOLOGÍA}

El método empleado fue el DACUM, que se caracteriza por una técnica de enfoque cualitativo que permite detectar funciones, habilidades, actitudes y conocimientos de un determinado cargo basado en la realización de un grupo focal. El producto de la 
investigación consiste en un gráfico de actividades de "página única" que puede tomarse como diagnóstico funcional.

La metodología DACUM se llevó a cabo en cuatro etapas: la primera, llamada definición del alcance del análisis, consistió en una investigación profunda sobre la amplitud de la ocupación a investigar. En esta etapa fueron identificados y definidos los profesionales para actuar en distintos contextos en enfermería. El estudio de alcance del área de actuación del enfermero se realizó entre el veinte de junio y el seis de julio del 2005, consultando una extensa lista de especialistas de instituciones públicas y privadas. En esta etapa se conocieron las diversas posibilidades de actuación y de inserción del enfermero en el mercado laboral. En la definición de alcance del análisis fue seleccionada una comisión compuesta por enfermeros especialistas en distintas áreas para el mapa de funciones y competencias. La comisión fue escogida con la intención de cubrir la mayoría de las categorías o puestos de trabajo pertenecientes a enfermería. Así, se pudieron combinar profesionales que actuaban en asistencia y gestión, consideradas extensas áreas de trabajo del enfermero. Para la selección de los sujetos de la investigación fueron considerados los siguientes criterios: tener entre cinco y diez años de experiencia, estar empleado exclusivamente en la ocupación y ser considerado como referente por sus pares.

La segunda etapa se llevó a cabo después de la definición de un facilitador con conocimiento de metodología DACUM y comprometido con el proceso de preparación y descripción de la ocupación. También se definió un relator que registró las funciones y competencias en tarjetas de forma clara y concisa durante el proceso de análisis. En la secuencia se realizó la reunión de descripción. En base al resultado de la investigación de alcance sobre las principales áreas de enfermería fueron definidas instituciones de naturaleza pública y privada, enfermería hospitalaria, salud colectiva, docencia de curso técnico, auditoría y atención pre-hospitalaria de Belo Horizonte - MG en 2005. En esta etapa, los sujetos fueron 11 enfermeros que suministraron información sobre las características del trabajo, las grandes áreas de competencias, las funciones, habilidades, actitudes y conocimientos empleados en el día a día. La pregunta a los enfermeros investigados y base de la descripción de las grandes áreas de competencias técnicas fue: “¿Qué haces?” Para describir las actitudes, habilidades y conocimientos se hizo la siguiente pregunta: "Para realizar todas las grandes áreas de competencia técnica, debes demostrar capacidad de.....".

En la tercera etapa se efectuó la reunión de validación con miras a confirmar lo que se había construido en la reunión de descripción. En esta etapa del análisis participaron 7 enfermeros con características similares a las del primer grupo. El proceso para validar el documento en base a los relatos de los sujetos dio prioridad a identificar las posibles lagunas en relación a los perfiles de los participantes e investigar profesionales capaces de cubrirlas.

La cuarta etapa de aplicación del método DACUM consistió en la reunión de conclusión, donde se identificaron los perfiles por actividades y se conocieron las áreas de conocimiento, así como también se relevaron los datos recogidos para finalizar la construcción de un informe orientado por los análisis del facilitador.

Cabe subrayar que el estudio fue aprobado por el Comité de Ética en Investigación de Seres Humanos de la UFMG, respetando los principios éticos de la investigación, en conformidad con la Resolución CNS 196/196 ${ }^{(5)}$. 


\section{RESULTADOS Y DISCUSIONES}

Como resultado de la primera etapa se lograron identificar las instituciones en las cuales el enfermero actúa con más frecuencia, como se resume en la Tabla I.

Tabla I- Relación entre línea de trabajo y especialidades encontradas en el mercado actual según el tipo de institución en el año 2005- Belo Horizonte - MG

\begin{tabular}{l|l}
\hline Línea de trabajo & Instituciones \\
\hline & Hospital (Centro Quirúrgico, Terapia Intensiva, Nefrología, \\
& Neonatología, Control de Infección Hospitalaria, \\
Cardiovascular, Endoscopía, Oftalmología, Oncología, \\
Obstetricia y Ginecología, Banco de Leche Humana, \\
Clínica Quirúrgica, Dermatología, Diagnóstico por imagen, \\
Endocrinología, Estomaterapia, Hemodinámica, Apoyo \\
Nutricional, Otorrinolaringología, Pediatría, Traumato- \\
ortopedia, Trasplante, Bioticista).
\end{tabular}

Salud colectiva - Pública (Salud Pública, Salud de la Familia, Sexología Humana, Asistencia al Adolescente, Campo Asistencial $\quad$ Educación en enfermería, Enfermedades Infecciosas).

\section{Hospital/Cersam/Centro de Convivencia/Viviendas Asistidas (Enfermero Psiquiátrico/Salud Mental).}

Atención préhospitalaria/SAMU (Enfermero de abordo/aeroespacial, Home Care / Domiciliaria, Empresas privadas).

Asilos y guarderías (Gerontología, geriatría y Pediatría). Empresas de asistencia pre-hospitalaria aeroespacial Representante médico

Empresas/SAST (Enfermero del trabajo e Informática)

Otros (Ética y Bioética, Terapias naturales, tradicionales y complementarias/no convencionales

Campo Gerencial

Hospital/Secretarías/Distritos/Unidades Funcionales

(Auditoría, Gerencia hospitalaria, Gerencia de unidad básica de salud, Gerencia de sectores (distrito/hospital/secretarías)).

Fuente: Elaborado para fines de este estudio

De acuerdo con las informaciones recogidas en la investigación de alcance, en enfermería tienden a crecer actividades fuera del hospital (enfermero domiciliario, atención domiciliaria); geriatría y/o gerontología; representación de medicamentos; gerencia y en el campo de la bioética. Cabe destacar que, durante los últimos años, la población brasileña ha vivido un franco proceso de transición demográfica debido a la caída de la tasa de mortalidad general y a la tasa de fecundidad cuya consecuencia recae en el aumento de esperanza de vida y envejecimiento gradual de las personas. Hoy en día, aproximadamente el 8,6\% de los brasileños tienen más de 65 años de edad ${ }^{(6)}$; por lo tanto, los profesionales de salud 
precisan calificación específica para atender a las demandas de la población anciana, lo cual explica la búsqueda de capacitación en las áreas de geriatría y gerontología.

El desplazamiento de la prestación de cuidados a los enfermos en ambientes hospitalarios para promocionar la salud transfiriendo los cuidados al domicilio exige acciones puestas en práctica con la visita e internación domiciliaria. Las actividades involucradas en la asistencia domiciliaria incluyen educación, prevención, recuperación y mantenimiento de la salud de los individuos y sus familiares dentro del contexto de sus viviendas. Este tipo de asistencia supera las prácticas institucionalizadas de salud con miras a la construcción de una nueva acción profesional en base a la inserción de los profesionales de salud en el lugar donde hay vida, interacción y relaciones de los individuos en su comunidad y, principalmente, en su domicilio ${ }^{(7)}$. En este sentido, el enfermero debe ser apto para utilizar tecnologías blandas y proporcionar modos de cuidar más acogedores, incluyendo lazos de confianza entre profesionales, usuarios, familia y comunidad.

Las tecnologías en el área de la salud fueron agrupadas por Merhy ${ }^{(8)}$ en tres categorías: duras, que se refieren a los materiales de consumo, a los permanentes, a los equipamientos y mobiliarios. Las tecnologías blandas-duras están representadas por las materias que rigen la salud (epidemiología, clínica médica, etc.) y las tecnologías blandas por la producción de la comunicación, de las relaciones y vínculos que están involucradas en el proceso saludenfermedad. Estas tres categorías están estrechamente vinculadas y presentes en el día a día de la enfermería ${ }^{(9)}$.

Otra área de actuación mencionada por los enfermeros es la de representación de medicamentos, que refleja el reconocimiento de las competencias del enfermero por parte de los empleadores de dicho sector.

En lo que se refiere a la gestión, las empresas han demandado profesionales que respondan con agilidad a sus expectativas. Los servicios de salud, principalmente los hospitales, intentan adecuarse a los nuevos modelos de gestión ${ }^{(10)}$. Se destaca que las actividades relacionadas con la gerencia en el área de salud han sido históricamente incorporadas como función del enfermero ${ }^{(11)}$, quien debe prepararse adecuadamente para los cambios constantes de perfil de los gestores. Actualmente, entre otras características, se exige de los gestores flexibilidad, capacidad de relacionarse y coraje para enfrentar los nuevos desafíos $^{(10)}$. Específicamente, en relación a la gestión de personas, la búsqueda constante de calidad y excelencia en los servicios ha provocado cambios significativos en esta área, buscando invertir cada vez más en los individuos y en las relaciones humanas ${ }^{(10)}$. Este modelo ha hecho que los enfermeros intentasen especializarse en las áreas de gestión y administración, ocasionando un crecimiento sustancial en este campo de actuación ${ }^{(12)}$.

En cuanto a la actuación del enfermero en el área de la Bioética, ésta refleja el momento actual en el cual son relevantes los problemas éticos resultantes de la evolución en el perfil de los pacientes, de las enfermedades, de los nuevos modelos asistenciales y de las tecnologías en salud. También se ponen en evidencia otras cuestiones referentes al inicio y final de la vida humana, nuevos métodos de fecundación, selección de sexo, ingeniería genética, maternidad sustitutiva, investigación en seres humanos, trasplante de órganos, pacientes terminales, entre otros (13). La International Association of Bioethics define el término Bioética, como: "estudios de los aspectos éticos, sociales, legales, filosóficos y de otros aspectos afines, inherentes a la asistencia médica y a las ciencias biológicas" (14). Así, el enfermero convive permanentemente con estos asuntos en su labor cotidiana, debiendo dominar aspectos éticos para poder tomar decisiones reflexivas, efectivas y seguras. El comportamiento ético es crucial para que el enfermero sea capaz de tomar decisiones que 
intenten promocionar la dignidad humana y la calidad de vida, tanto en la práctica del cuidado como en la investigación ${ }^{(15)}$.

Con relación al perfil de los sujetos de estudio, obtenidos en la segunda etapa de este trabajo, las áreas de actuación señaladas fueron amplias y variadas, desde la hospitalaria (CTI, quirúrgico, neonatología, control de material, clínica médica, trasplante, nefrología, dermatología, urgencias, pediatría, obstetricia etc.), salud colectiva (PSF, salud mental, enfermería del trabajo, asilos y guarderías), auditoría, prehospitalaria, docencia de curso técnico hasta gestión. Fue mencionado que, a pesar de no haber habido participación directa de profesionales del PSF ni de investigadores en la reunión de descripción, en la etapa de validación esa laguna fue cubierta por la gran experiencia de los dos grupos. El tiempo promedio de actuación en enfermería es de diecinueve años.

En cuanto al número de empleos, la mayoría de los enfermeros tenía dos tipos de trabajo: formal o informal. Dentro de una perspectiva amplia, se puede deducir que esa situación refuerza la caracterización del trabajo del enfermero como intenso y, al mismo tiempo, de remuneración insuficiente o factible de complementación ${ }^{(16)}$. En lo referente a la cualificación profesional, los sujetos del estudio mostraron un alto nivel de educación formal, incluyendo cualificación educacional y experiencia en diversas áreas de enfermería durante su trayectoria profesional.

La tercera etapa de la investigación consistió en el relato de los enfermeros acerca de sus actividades cotidianas mediante preguntas discutidas en el grupo focal. Los resultados están en las tablas que indican también los despliegues de cada gran área de competencia técnica relatada y sus subactividades, siempre orientadas por la matriz DACUM.

La primera gran área de competencia se refiere a la asistencia al cliente, usuario, familia, comunidad y trabajador (Tabla II).

[Tabla II - Descripción de la gran área de competencia (A) y sus subactividades - EEUFMG, Belo Horizonte, 2005]
Gran Área de Competencia GAC - A

Asistir al cliente/usuario/familia/ Comunidad/trabajador

\section{Subactividades}

A1-Realizar la acogida/consulta/anamnesis de enfermería

A2- Realizar una evaluación sistemática de la evolución del cliente

A3- Realizar un diagnóstico y prescripción de los cuidados de enfermería

A4- Prestar cuidados de enfermería de baja, mediana y alta complejidad

A5- Registrar procedimientos, observaciones y cuidados de enfermería

A6- Prescribir medicación de acuerdo con protocolos institucionales

A7- Brindar apoyo emocional/espiritual al paciente/familia

A8- Acompañar al transporte inter/intrahospitalario

A9- Atender a la familia del paciente/trabajador/cuidador

A10-Visitar al cliente en el domicilio

A11- Acompañar a los clientes en los programas de atención domiciliaria

A12- Acompañar a los clientes en las actividades 
externas/talleres terapéuticos

A13- Acompañar al equipo multidisciplinario en procesos de salud

Fuente: DACUM - Gran área de competencia A - de los enfermeros participantes del "Proyecto de Cambio Curricular de la EEUFMG de 2005”.

La competencia relacionada con la asistencia al cliente, usuario, familia, comunidad y trabajador trata de la enfermería como práctica social, política e históricamente construida con la finalidad de cuidar al ser humano en todas las etapas de la vida. Contribuye, también, a promover, prevenir, recuperar y rehabilitar la salud ${ }^{(17)}$. En este estudio el cuidado es el foco y lo principal porque se refiere a las acciones y actividades orientadas a dar asistencia, apoyo o capacitación a individuos o grupos con necesidades evidentes o previstas, buscando mejorar la condición o modo de vida humana ${ }^{(18)}$.

En el campo de la enfermería los objetos de trabajo son el cuidado de enfermería y su gestión. Este cuidado se concibe como un conjunto de acciones de seguimiento continuo del usuario/población, durante las enfermedades o a lo largo de procesos socio vitales (salud del niño, adolescente, adulto, mujer, anciano, trabajador, etc.), buscando la promoción, prevención y recuperación de la salud ${ }^{(19)}$.

La asistencia trata de la capacidad de poner en práctica las acciones y conocimientos y su objetivo es el cuidado demandado por individuos, familias, grupos sociales, comunidades y colectividades. Por tratarse de asistir al ser humano estos cuidados son de naturaleza física, psíquica, social y espiritual en todas las etapas de la vida.

El enfermero tiene permiso legal para practicar la asistencia así como para delegar funciones compatibles con su nivel de instrucción al técnico de enfermería. Para ello se exigen conocimientos, habilidades y actitudes relacionados a las tomas de decisión, así como a la elaboración, ejecución y evaluación de los planes asistenciales.

Como en otros procesos laborales, el de enfermería no es unívoco, en el sentido de la existencia de un solo objeto demandando finalidades, medios e instrumentos. Al contrario, se pueden identificar distintos procesos entre los cuales los más conocidos son asistir/cuidar, administrar, investigar y calificar y re-calificar el equipo ${ }^{(20)}$.

[Tabla III - Descripción de la gran área de competencia (B) y sus subactividades EEUFMG, Belo Horizonte, 2005].

\begin{tabular}{l|l}
\hline Gran Área de & Subactividades \\
Competencia - & \\
GAC - B &
\end{tabular}

B1- Definir atribuciones

B2- Delegar funciones

B3- Seleccionar los recursos humanos

B4- Dimensionar los recursos humanos

B5- Elaborar escalas de servicios/reorganización

Coordinar Equipos de B6- Reunirse con equipos de trabajo

Trabajo

B7- Estandarizar normas, procedimientos y rutinas de enfermería

B8- Participar de la elaboración de

protocolos/manuales

B9- Monitorear el proceso laboral

B10- Evaluar el desempeño del equipo de trabajo 
B11- Aplicar medidas administrativas

B12- Aplicar dinámicas de grupo

B13- Promover eventos de socialización

Fuente: DACUM - Gran área de competencia B - de los enfermeros participantes del

"Proyecto de Cambio Curricular de la EEUFMG de 2005".

La Tabla III muestra las actividades administrativas como inherentes a la coordinación de equipos. En 1986, por medio de la Ley n. $7498^{(21)}$, que dispone sobre la reglamentación del ejercicio de la enfermería en Brasil, se formalizó el derecho privado del enfermero de desempeñar actividades relacionadas a la "planificación, organización, coordinación, ejecución y evaluación de los servicios de asistencia de enfermería". Las competencias específicas para ejercer tareas de administración de enfermería y de los servicios de salud propuestas por las DCNENF encuentran eco en los resultados de este estudio, pues la competencia de coordinar equipos de trabajo está aliada a los conocimientos del área administrativa y de gestión. Esto se debe a que el enfermero ocupa cada vez más espacio en la gestión hospitalaria, ya sea en la gestión de unidades operativas o asumiendo cargos administrativos que exigen una visión más amplia del funcionamiento general de la organización, sobre todo conocimiento en gestión de personas y de recursos financieros, físicos, materiales, tecnológicos ${ }^{(12)}$.

En la enfermería, según Greco ${ }^{(22)}$, la gerencia consiste en la previsión, provisión, mantenimiento, control de recursos materiales y de personas para el funcionamiento del servicio. La gerencia del cuidado, sin embargo, consiste en el diagnóstico, planificación, ejecución y evaluación de la asistencia, pasando por la delegación de las actividades, supervisión y orientación del equipo. Las demandas actuales están directamente relacionadas no sólo con la formación técnica de los enfermeros, sino también con el desarrollo de la competencia interpersonal, herramienta indispensable para transformar la práctica de gestión del enfermero ya que se constituye de un conjunto de aptitudes y actitudes adquiridas y vinculadas entre sí ${ }^{(23)}$.

Otra gran área de competencia señalada en este estudio está vinculada a la capacidad de desarrollar educación en salud (Tabla IV).

[Tabla IV - Descripción de la gran área de competencia (C) y sus subactividades EEUFMG, Belo Horizonte, 2005].

\section{Gran Área de Competencia -GAC - C}

C1- Relevar la necesidad/demanda de entrenamiento

C2- Definir los temas/objetivos

C3- Definir el público objeto

C4- Prever los recursos materiales y financieros

Desarrollar Educación en Salud
C5- Definir el cronograma

C6- Preparar el material didático

C7-Impartir los cursos técnicos, charlas,

seminarios/entrenamiento

C8- Efectuar el seguimiento de pasantías de nível medio/superior

C9- Moniteorar el programa de educación

C10- Formar el grupo operativo/educativo

C11- Orientar a la comunidad

C12- Determinar una estrategia metodológica de

enseñanza 
Fuente: DACUM - Gran área de competencia C - de los enfermeros participantes del "Proyecto de Cambio Curricular de la EEUFMG de 2005".

Las actividades de educación demandadas en enfermería están, en su mayoría, orientadas a la realización de cursos con énfasis en la formación profesional. La enfermería es compleja porque la finalidad social de su profesional exige el dominio de un conjunto de conocimientos generales y especializados que se deben autoalimentar, desarrollar y garantizar en el progreso técnico y tecnológico que requiere la profesión ${ }^{(3)}$. Al asumir cargos de supervisor del equipo de enfermería, el enfermero demuestra habilidad de interactuar con los demás elementos del equipo. El trabajo de enfermería se caracteriza por un equipo de profesionales con competencias y atribuciones diferenciadas, con capacitación de nivel medio y superior. Debido a la necesidad de prestar cuidados calificados en los servicios de salud, al enfermero le corresponde detectar la falta de orientación y necesidad de desarrollo de los empleados. Así, el foco de la gestión de las personas dejó de ser el control y pasa a estar orientado al desarrollo del equipo de los trabajadores/ miembros del equipo ${ }^{(24)}$.

En este estudio el desarrollo de la Educación en Salud se confunde con subactividades para la educación permanente. Cabe aclarar que la primera se constituye en una práctica social y transformadora en la cual la población participa en el contexto de su vida cotidiana con sus propios problemas de salud ${ }^{(25)}$. La segunda se caracteriza por tener un público-objeto multiprofesional, dirigirse a una práctica institucionalizada, enfocar los problemas de salud y tener como objetivo la transformación de las prácticas técnicas y sociales, ser de periodicidad continua, utilizar metodología centrada en la resolución de problemas y buscar el cambio ${ }^{(26)}$ como resultado. Se observa que, al asumir el compromiso con la educación y entrenamiento/pasantías de las futuras generaciones profesionales, los enfermeros están atendiendo a los dictámenes de las DCNENF.

Los sujetos del estudio identificaron también la capacidad para administrar recursos materiales y equipamientos, como indica la Tabla $\mathbf{V}$.

[Tabla V - Descripción de la gran área de competencia (D) y sus subactividades - EEUFMG, Belo Horizonte, 2005].

\begin{tabular}{l|l}
\hline Gran Área de & Subactividades \\
Competencia - & \\
GAC - D & \\
\hline
\end{tabular}

\begin{tabular}{l|l}
\hline & D1- inventariar material y equipamiento \\
& D2- estandarizar el material/equipamiento \\
& D3- describir el perfil físico, químico y biológico del \\
Administrar Recursos & producto \\
M4- prever el material y equipamiento \\
D5- presupuestar el material/equipamiento \\
& D6- analizar el costo-beneficio del material médico- \\
& hospitalario \\
& D7- evaluar la calidad de material/equipamiento \\
& D8- procesar el material médico-hospitalario \\
& D9- participar de los procesos de licitación \\
& D10- prover el material y equipamiento \\
& D11- controlar el flujo de material y equipamiento \\
\hline
\end{tabular}

Fuente: DACUM - Gran área de competencia C - de los enfermeros participantes del "Proyecto de Cambio Curricular de la EEUFMG de 2005". 
La competencia de administrar recursos materiales y equipamientos exige actividades que incluyen desde conocimiento sobre nuevas tecnologías hasta su adquisición, control y comprobación de calidad. Los avances tecnológicos en las instituciones de salud aunados a la necesidad de disminuir costos exigen de los profesionales un nivel de desarrollo mayor en lo que se refiere a la administración de los recursos materiales. Realzamos que los gastos en relación a estos recursos representan una importante porción del presupuesto de las organizaciones ${ }^{(27)}$. Los sistemas de recursos materiales de las organizaciones hospitalarias han registrado entre tres y seis mil productos de consumo.

Para Chiavenato ${ }^{(28)}$, la gestión de recursos materiales involucra la totalidad de los flujos de material de una organización, programación, compra, recepción, almacenamiento en el depósito, desplazamiento, transporte interno y almacenamiento. Esta gran área de competencia en el campo de la enfermería adquiere mucho sentido porque el gerente de unidades asume la coordinación de toda la actividad asistencial, con un rol preponderante en cuanto a la determinación del material necesario para la ejecución de las actividades ${ }^{(29)}$. Entonces, le corresponde al enfermero conocer y acompañar el perfil de los gastos de productos de la unidad, no sólo los de consumo sino también de medicamentos y artículos esterilizados. Es fundamental, luego, que el enfermero se mantenga actualizado sobre los nuevos productos, nuevas técnicas terapéuticas y nuevas formas de capacitación de los profesionales para usar con racionalidad los productos disponibles. La actuación del enfermero en la gestión de recursos materiales es un importante papel en la dimensión técnico administrativa inherente a los procesos de cuidar y administrar ${ }^{(29)}$.

Las actividades de planificar acciones de salud componen otra gran área de competencia del enfermero (Tabla VI).

[Tabla VI - Descripción de la gran área de competencia (E) y sus subactividades EEUFMG, Belo Horizonte, 2005].

\begin{tabular}{l|l}
\hline Gran Área de & Subactividades \\
Competencia - & \\
GAC - E & \\
\hline
\end{tabular}

\section{Competencia - GAC - E}

Planificar acciones de salud

\author{
E1- Realizar el diagnóstico situacional \\ E2- Identificar el perfil del público objeto \\ E3- Establecer las prioridades \\ E4- Establecer las metas/indicadores \\ E5- Definir el proceso terapéutico \\ E6- Formular los instrumentos y métodos de trabajo \\ E7- Organizar el ambiente para procesos \\ asistenciales/trabajo \\ E8- Elaborar un plan de acción
}

Fuente: DACUM - Gran área de competencia C - de los enfermeros participantes del "Proyecto de Cambio Curricular de la EEUFMG de 2005".

Con respecto a la planificación de las acciones de salud, mencionamos que la ley del ejercicio profesional de enfermería establece como prioridad del enfermero la participación en la elaboración, ejecución y evaluación de la programación de salud. Observando las subactividades citadas (Tabla VI), notamos que la planificación de las acciones de salud es un proceso dinámico, siendo necesario considerar la realidad de los diferentes servicios de salud y de los individuos involucrados en las diferentes situaciones. En ese sentido, se puede definir la planificación como el arte de hacer elecciones y de elaborar planes para 
favorecer un proceso de cambio ${ }^{(30)}$. La planificación se ha vuelto un tema cada vez más importante en la vida del hombre contemporáneo de tres maneras: como instrumento de los procesos de gestión de las organizaciones, como impulsor de prácticas sociales transformadoras y como método de acción gubernamental ${ }^{(31)}$.

Actualmente, las organizaciones de salud buscan concretar sus objetivos sin perder de vista la calidad en el servicio. La eficacia de dichas organizaciones está sujeta a las relaciones que establecen personas, tecnologías, recursos y administración ${ }^{(32)}$. La aplicación de estos factores depende, sin embargo, de las competencias, habilidades y conocimientos de los profesionales sobre el aprovechamiento y los métodos de planificación para entender su aplicabilidad en los diferentes servicios de salud.

En la gran área de competencia relacionada con la vigilancia en salud (Tabla VII), se observa el desmembramiento de funciones investigativas, de control, orientación y evaluación que, de cierta forma, logran -aunque no sea la prevención- al menos atenuar problemas vinculados a los riesgos que pueden afectar la salud del individuo. A ese respecto se subraya que la vigilancia en salud articula epidemiología, planificación y organización de los servicios de salud y la comprensión de las desigualdades sociales como determinantes de la distribución desigual de los agravios sobre la población. Pone en práctica tres tipos de acciones de salud: promoción, prevención de enfermedades y accidentes y atención colectiva $^{(33)}$.

[Tabla VII - Descripción de la gran área de competencia (F) y sus subactividades EEUFMG, Belo Horizonte, 2005].

\begin{tabular}{l|l}
\hline Gran Área de & Subactividades \\
Competencia - & \\
GAC - F &
\end{tabular}

F1-Relevar los datos socioeconómicos
demográficos/ epidemiológicos (realizar búsqueda
activa)
F2- Investigar los factores de riesgo a la salud
F3- Investigar el nexo epidemiológico/causal
F4- Identificar el portador de sufrimiento
mental/agravamiento ocupacional
F5- Notificar las enfermedades compulsorias
F6- Notificar los accidentes de trabajo
F7- Evaluar la exposición al riesgo ocupacional
F8- Evaluar la capacidad funcional del trabajador
F9- Orientar las medidas de prevención y control de
infecciones hospitalarias/bioseguridad

Realizar Vigilancia en Salud

Fuente: DACUM - Gran área de competencia C - de los enfermeros participantes del "Proyecto de Cambio Curricular de la EEUFMG de 2005".

La vigilancia en salud puede entenderse como la redefinición de las prácticas sanitarias y considera dos dimensiones: la técnica, que concibe la vigilancia de la salud como modelo asistencial alternativo compuesto por un conjunto de prácticas destinadas a controlar determinantes, riesgos y daños y la dimensión administrativa, que concibe la vigilancia de la salud como práctica que organiza el trabajo en salud de modo que enfrente problemas en un determinado territorio ${ }^{(34)}$. Las dimensiones orientadas a la vigilancia en salud coinciden con dos grandes vertientes del proceso de trabajo del enfermero: la asistencia y la gestión, reforzando la relación de este profesional con los determinantes sociales del proceso salud- 
enfermedad, con el desarrollo de prácticas de prevención, promoción y recuperación de la salud de los grupos sociales, principalmente en el área de salud colectiva.

El área de competencia vigilancia en salud no se limita a las prácticas de salud que sólo consideran la población sino que incluye el cuidado al trabajador del área de salud. La vigilancia en salud del trabajador se distingue de la vigilancia en salud en general y de otras disciplinas en el campo de las relaciones entre salud y trabajo por delimitar como su objeto específico a la investigación e intervención en la relación del proceso de trabajo con la salud $^{(35)}$. Según las subactividades citadas, la vigilancia en salud del trabajador se puede entender como la articulación entre controles de exposición y efectos. En esta gran área de competencia lo que se destaca es el resultado de las discusiones entre las instituciones y trabajadores sobre las diferentes formas de exposición de los trabajadores en el ambiente laboral.

[Tabla VIII - Descripción de la gran área de competencia (G) y sus subactividades EEUFMG, Belo Horizonte, 2005].

\begin{tabular}{l|l}
\hline Gran Área de & Subactividades \\
Competencia - & \\
GAC - G & \\
\hline
\end{tabular}

G1- Elaborar una planilla de costos

G2- Analizar los indicadores de costos/facturación

G3- Seguir el flujo financiero

G4- Captar los recursos financieros

Administrar Recursos $\quad$ G5- Proponer las directrices para la reducción de

Financieros

costos/aumento de recetas

G6- Proponer las directrices para la reducción de

costos/aumento de recetas

G7- Completar el formulario de autorización para los

procedimientos de alto costo/producción

G8- Participar de las decisiones sobre la asignación de

los recursos financieros

Fuente: DACUM - Gran área de competencia C - de los enfermeros participantes del "Proyecto de Cambio Curricular de la EEUFMG de 2005".

Observando las subactividades descritas en esta área de competencia verificamos que los conocimientos relacionados con los recursos financieros se han transformado en una nueva área del conocimiento en la enfermería (Tabla VIII). Actualmente, el gran reto de las instituciones de salud es atender las demandas de sus servicios con calidad a bajos costos. En ese sentido, el enfermero se enfrenta con la necesidad de conocer su producción y de relacionarla con el perfil de gastos y análisis de costos, porque la mejora de los servicios de salud depende de los recursos disponibles y de su aplicación. Desde 1993 el Consejo Internacional de Enfermería afirma que las finanzas constituyen otra área de dominio del conocimiento en enfermería y recomienda que los profesionales realicen estudios de investigación para validar metodologías de cálculo de costos en los servicios de enfermería (36)

El enfermero está ocupando cada vez más cargos administrativos en las unidades de salud. Es responsable de la gestión de personas, recursos materiales y físicos que consumen gran volumen de recursos financieros ${ }^{(37,12)}$. Se da por entendido, gestión de costos en enfermería: "El proceso administrativo que busca el control de costos y la toma de decisión de los enfermeros para una racionalización eficiente de recursos disponibles y limitados, con el objetivo de alcanzar resultados coherentes con las necesidades de salud de la clientela y con las finalidades institucionales" (38). 
El control de costos es esencial para que los enfermeros y gerentes de unidad de salud puedan seguir la evolución de su servicio mejorando el desempeño, redefiniendo prioridades, racionalizando recursos y siguiendo la productividad ${ }^{(36)}$.

Considerando la participación del enfermero en la elaboración de políticas de salud y en la evaluación de la calidad de dichos servicios, le corresponde al enfermero prestar consultoría y auditoría en salud (Tabla IX).

[Tabla IX - Descripción de la gran área de competencia $(H)$ y sus subactividades EEUFMG, Belo Horizonte, 2005].

\section{Gran Área de Competencia - GAC - H}

Prestar

consultoría/auditoría en salud

\section{Subactividades}

H1- participar de la elaboración de políticas de
salud
H2- participar de la elaboración de proyectos del
área física en los establecimientos de salud
H3- asesorar en la implantación y
operacionalización de los servicios de salud
H4- emitir los pareceres técnicos
H5- producir los informes técnicos
H6- participar de las comisiones /colegiados
/consejos /conferencias
H7- participar de la formulación de pruebas/testes
para oposiciones
H8-evaluar la calidad de los servicios (técnicas,
procedimientos, exposición al riesgo)
H9- verificar las anotaciones en las historias
clinicas
H10- evaluar la cuenta hospitalaria
H11- encaminar el registro para la corrección
H12- evaluar el informe de exclusión
H13- presentar recursos de las exclusiones
H14- negociar con el auditor externo
H15- promover la venta de
medicamentos/material y el equipamiento
médico-hospitalario

Fuente: DACUM - Gran área de competencia C - de los enfermeros participantes del "Proyecto de Cambio Curricular de la EEUFMG de 2005".

Las subactividades expresadas en la Tabla IX refuerzan el hecho de que la auditoría es una de las responsabilidades de los enfermeros investigados y de que agrupa una serie de actividades políticas y de negociación. Actualmente, en el sector de la salud, todas las actividades dedicadas a la calidad de los servicios y al área contable están en expansión. Entre estas actividades la auditoría de Enfermería está insertada en la auditoría en salud permitiendo analizar las cuestiones específicas de enfermería. En la auditoría de enfermería se llevan a cabo acciones que buscan la calidad de la asistencia, con reducción de costos, agregando valores financieros a los valores cualitativos ${ }^{(39)}$. Según la misma autora, la auditoría en enfermería es la evaluación sistemática de la calidad de la asistencia de la enfermería. La percepción del enfermero como participante en el proceso de auditoría remite 
a una asistencia eficiente, pues estos profesionales dominan el conocimiento de los procesos administrativos y beneficios de los servicios para una asistencia de calidad.

En la auditoría, el enfermero tiene la posibilidad de desarrollar indicadores de asistencia, establecer criterios de evaluación y, por consiguiente, generar nuevos conocimientos que se obtienen identificando los problemas de enfermería, las diversas conductas adoptadas y el grado de resolutividad ${ }^{(39)}$. De esta manera, el servicio de salud puede contar con más beneficios $y$, consecuentemente, menos riesgos a los pacientes. En esta área de competencia percibimos la aproximación entre auditoría y consultoría en relación a las actividades de elaboración de proyectos, lo cual puede tener que ver con la necesidad de buscar soluciones pertinentes para el buen funcionamiento del servicio. Realzamos que la práctica del enfermero en el área de auditoría está reglamentada por el Consejo Federal de Enfermería por medio de la resolución $266^{(40)}$, que dispone sobre la organización, dirección, coordinación, evaluación, prestación de consultoría, auditoría y emisión de pareceres sobre los servicios de auditoría en enfermería.

[Tabla X - Descripción de la gran área de competencia (I) y sus subactividades - EEUFMG, Belo Horizonte, 2005].

\section{Gran Área de Competencia - GAC - I}

Actuar en investigación en el área de la salud

\section{Subactividades}

11- Revisar la literatura

12- Definir la metodología de investigación

13- Analizar los proyectos de investigación

14- Dar apoyo logístico en trabajos de campo

15- Recoger datos cuantitativos y cualitativos

16- Analizar datos

17- Producir artículos

18- Tratar datos

19- Someter trabajos científicos a publicación

Fuente: DACUM - Gran área de competencia C - de los enfermeros participantes del "Proyecto de Cambio Curricular de la EEUFMG de 2005".

Las subactividades relatadas en la competencia sobre investigación en el campo de la salud reflejan las dificultades para discutir la investigación en la enfermería (Tabla $\mathbf{X}$ ). Según Carvalho $^{(41)}$, en Brasil la mayoría de los enfermeros está dando los primeros pasos en la investigación científica. La diseminación de los estudios de investigación en el campo de la salud ha sido estimulada por la constatación de que las profesiones sólo solidifican un cuerpo de conocimiento con la producción científica ${ }^{(42)}$. Así, es fuerte la discusión sobre la inclusión de contenidos, disciplinas y estrategias que estimulen la investigación en las universidades. Por ser considerada una profesión nueva, la enfermería precisa agregar nuevos conocimientos para ampliar el pilar teórico-práctico. La investigación es una práctica cada vez más común que ocurre en diferentes escenarios y niveles ${ }^{(42)}$.

Es fundamental que la enseñanza de grado incluya en todo su proceso actividades vinculadas a la investigación para formar sujetos críticos y creativos en su práctica profesional. Para ello, la investigación debe ser la esencia de la práctica educativa en enfermería ya que se observa "una estrecha relación entre la formación de un profesional crítico-creativo y la actitud investigativa" ${ }^{(43)}$. 


\section{CONSIDERACIONES FINALES}

El curso de enfermería de la EE-UFMG revisa constantemente los procesos de trabajo del enfermero a causa de los cambios constantes del modelo demográfico de la población, del perfil epidemiológico y de los estilos de vida, entre otros, que imponen nuevas exigencias a todos los trabajadores del área de salud.

La realización de este estudio ha permitido percibir que en la enfermería como práctica social hay procesos de trabajo singulares y distintos con competencias enfocadas en acciones asistenciales, administrativas, de educación y de investigación, que coinciden con aquéllas establecidas por las DCNENF.

Destacamos que las escuelas de enfermería han enfocado el desarrollo de planes de estudio orientados a la formación por competencias. En este sentido, el presente estudio de investigación ha permitido utilizar una estrategia para construir el Proyecto Pedagógico con miras a la tan anhelada formación, principalmente, articulada con la realidad de los servicios. Desde esa perspectiva, se puede deducir que, con una formación basada en competencias el alumno estará mejor calificado para actuar en armonía con las demandas sociales y del mercado.

La metodología Developing a Curriculum ha permitido reconocer las competencias que el enfermero necesita para enfrentar varias áreas de actuación y, principalmente, ha permitido la participación colectiva de profesores, alumnos y enfermeros de los servicios en la construcción del Proyecto Pedagógico integrando enseñanza, trabajo y escuela.

Se realza la pertinencia y relevancia del uso de metodologías diferenciadas en la construcción de proyectos pedagógicos teniendo en cuenta la ampliación de perspectivas y las posibilidades de develar los innumerables escenarios de práctica en los cuales actúan los enfermeros.

\section{REFERENCIAS}

1- Brasil. Ministério da Educação. Diretrizes Curriculares Nacionais dos Cursos de Graduação em Enfermagem. Parecer CNE/CES. 1133/2001. Aprovado em 07/08/2001.

2- Pacheco J, Paraskeva J, Silva A. Reflexão e Inovação Curricular. Actas do III Colóquio sobre Questões Curriculares.1998

3- Dias MFPB. Construção e Validação de um Inventário de Competências: Contributos para a definição de um perfil de competências do enfermeiro como grau de licenciado. Editora: Lusociência, Portugal, 2006. 398 p.

4- Coffin L. Developing a Curriculum Facilitator Manual. The Canadian vocational Association, 1993.

5- Brasil. Conselho Nacional de Saúde. Resolução no 196 de 10 de outubro de 1996. Aprovadas diretrizes e normas regulamentadoras de pesquisas envolvendo seres humanos [Internet]. [Acesso em 15/07/08]. Disponível em: http://conselho.saude.gov.br

6- Brasil. Ministério da Saúde. Secretaria de Vigilância em Saúde. Departamento de Análise de Situação de Saúde. Uma análise da situação de saúde - Brasil, 2006b; 364p

7- Giacomozzi CM, Lacerda MR. A prática da assistência domiciliar dos profissionais da estratégia de saúde da família. Texto contexto Enf. Florianópolis, Out-Dez. 2006 15(4): 645653

8- Merhy EE, Chakkour M, Stéfano E, Santos CM, Rodrigues RA e Oliveira PCP. Em busca de ferramentas analisadoras das tecnologias em saúde. A informação e o dia a dia de um 
serviço, interrogando e gerindo trabalho em saúde. In: Agir em Saúde. Um Desafio para o Público (E. E. Merhy \& R. Onocko, org.).São Paulo: Editora Hucitec. 1997;113-150.

9- Barra DCC, Nasciment ERP, Martins JJ, Albuquerque GL, Erdmann AL. Evolução histórica e impacto da tencologia na área da saúde e da enfermagem. Rev. Eletr. de Enf. 2006 8(3): 422-30 [Internet]. [Acesso em 30/08/08]. Disponível em: http//:www.fen.ufg.br/revista/revista8 3/v8n3a13.htm

10-Cunha ICKO, Ximenes Neto FRG. Competências gerenciais de enfermeiras: um novo velho desafio? Rev. Texto - Contexto Enf. Florianópolis, Jul - Set. 2006; 15(003): 479-82.

11-Peres AM, Ciampone MHT. Gerência e competências gerais do enfermeiro. Texto contexto Enf. Florianópolis 2006 15(6): 492-9.

12-Brito MJM. A configuração identitária da enfermeira no contexto das práticas de gestão em hospitais privados de Belo Horizonte. [Tese]. Belo Horizonte. Faculdade de Ciências Econômicas da Universidade Federal de Minas Gerais; 2004. 393p.

13-Zanatta JM, Boemer MR. Bioética - Um Ensaio Sobre sua Inserção nos Cursos de Graduação em Enfermagem. Revista Eletrônica de Enfermagem [Internet]. [Acesso em 30/08/08]. 2005; 7(3): 351-54. Disponível em: http://www.fen.ufg.br/Revista/revista7 3/revisao 01.htm.

14-Campbell A. A Bioética no século XXI. Coleção saúde, cidadania e bioética, editora Universidade de Brasília, 2000.

15-Balduíno A deFA, Labronici LM, Corrêa MEC e Mantovani MF. A Bioética no cenário da enfermagem. Online Brazilian Journal of Nursing [Internet]. [Acesso em 30/08/08] 2007; 16(1). Disponivel em: http://www.uff.br/objnursing

16-Almeida MCPde. A formação do Enfermeiro frente a reforma sanitária. Cad. Saúde Pública. 1986; 4(2): 505-510.

17-Garanhani ML et al. Princípios pedagógicos e metodológicos do currículo integrado de Enfermagem. In: Dellaroza MSG, Vannuchi MTO. O currículo integrado do curso de enfermagem da Universidade Estadual de Londrina: do sonho à realidade. Editora HUCITEC: São Paulo, 2005; 3: 35-57.

18-Leininger MM. Cultural care diversity and universality: a theory of nursing. New York: National League for Nursing Press, 1991. 432 p.

19-Peduzzi M, Anselmi ML. O processo de trabalho da enfermagem: a cisão entre o planejamento e a execução dos cuidados. Rev. Bras. de Enf. 2002 55(4): 392-8.

20-Egry EY. As transformações nos processos de trabalho da enfermagem em saúde coletiva: validando a nomenclatura dos objetos e dos instrumentos. Relatório CNPq, 2000.

21-Brasil. 26 de jun. 1986, Lei n. 7498/86. Dispõe sobre a regulamentação do exercício da enfermagem e dá outras providências. Diário Oficial da União, Brasília, 1986. Seção: 92735.

22-Greco RM. Ensinando a Administração em Enfermagem através da Educação em Saúde. Brasília (DF). Rev. Bras. de Enf. 2004; 57(4): 504-7.

23-Bezerra ALQ, Munari DB. Inclusão da Competência Interpessoal na Formação do Enfermeiro como Gestor. Brasília (DF). Rev. Bras. de Enf. 2004; 57(4):484-6

24-Dutra JS. Gestão de Pessoas: modelo, processos, tendências e perspectivas. São Paulo: Atlas, 2002: 210p.

25-Barroso MGT, Vieira NFC, Varela ZMdeV. Ensino de Educação em Saúde, interdisciplinariedade e políticas públicas. Rev. Bras. de Promoção da Saúde. Fortaleza, 2006; 19(003): 182-7.

26-Peres AM, Ciampone MHT. Gerência e competências gerais do enfermeiro. Rev. Texto contexto - Enf. Jul./Set. 2006; 15(3):492-9

27-Vecina Neto G, Reinhardt Filho W. Gestao de recursos materiais e de medicamentos. São Paulo, FSP da USP, 1998

28-Chiavenato I. Iniciação a administração de materiais. São Paulo: Makron Books; McGraw Hill, 1991. 167p 
29- Castilho V, Gonçalves VLM. Gerenciamento de recursos materiais. In: Kurcgant, P (org) Gerenciamento de Enfermagem. Rio de Janeiro. Ed. Guanabara Koogan; 2005. p.157-170.

30-Ciampone MHT, Melleiro MM. O planejamento e o processo decisório como instrumentos do processo de trabalho gerencial. In: Kurcgant, P (org) Gerenciamento de Enfermagem. Rio de Janeiro. Ed. Guanabara Koogan; 2005. p.37-53.

31-Merhy EE. Em busca da qualidade dos serviços de saúde: os serviços de porta aberta para a saúde e o modelo tecno-assistencial em defesa da vida (ou como aproveitar os ruídos do cotidiano dos serviços de saúde e colegiadamente reorganizar o processo de trabalho na busca da qualidade das ações de saúde). In: Cecílio LCO. Inventando a mudança na saúde. São Paulo: Hucitec, 1994. p. 117-60.

32-Junqueira LAP. Gerência dos serviços de saúde. Cad. Saúde Pública, 1990;6(3):247-259

33-Bertozolli RM, Fracolli LA. Vigilância à saúde: alerta continuado em saúde coletiva. Mundo Saúde. 2005;28(1):14-20

34-Teixeira CF, Paim JS, Vilabôas AL. SUS modelos assistenciais e vigilância da saúde. Inf. Epi.SUS:1998; 2(1):7-28.

35-Machado JMH. Processo de vigilância em saúde do trabalhador. Cad. Saúde Pública, Rio de Janeiro, 199713 Supll 2

36-Castilho V, Fugulin FMT, Gaidzinski RR. Gerenciamento de custos nos serviços de Enfermagem. In: Kurcgant, P (org) Gerenciamento de Enfermagem. Rio de Janeiro. Ed. Guanabara Koogan; 2005. p.171-183.

37-Brito MJM. O enfermeiro na função gerencial: desafios e perspectivas na sociedade contemporânea. [Dissertação]. Belo Horizonte. Escola de Enfermagem da Universidade Federal de Minas Gerais; 1998. 176p

38-Francisco IM, Castilho VA. A enfermagem e o gerenciamento de custos. Rev. Esc. Enfer. USP, 2002; 36(3): 240-4.

39-Kurcgant P. Administração em enfermagem. São Paulo: EPU, 1991

40-Conselho Federal de Enfermagem. Out de 2001, Resolução n-266, [Internet]. [acesso em 28/08/08].Disponível em : www.corenmg.org.br.

41-Carvalho V. Cuidando, pesquisando e ensinando: acerca de significados e implicações da prática da enfermagem. Rev. Latino-Am. Enf. Ribeirão Preto; 2004 12(5): 806-15

42-Camponogara S, Kirchhof ALC, Gelbcke FL, Magnago TSBdeS. Espaço do dialogo na pesquisa em enfermagem: relato de experiência sobre a fase de coleta de dados. Texto contexto - enfe. 2007 Out - Dez; 16(4): 762-8.

43-Reibnitz KS, Prado ML. Formação do profissional crítico-criativo: a investigação como atitude de (re)conhecimento do mundo. Texto Contexto Enf. 2003 Jan-Mar; 12 (1): 26-33.

ISSN 1695-6141

๑ COPYRIGHT Servicio de Publicaciones - Universidad de Murcia 